\title{
A global journal
}

We want Nature Human Behaviour to be a platform for important science, comment and opinion from around the globe. To achieve this, we need your help.

$\mathrm{n}$ this issue, John Green Otunga, program coordinator of the UnaHakika Project and the Sentinel Project in Kenya, discusses a topic of significant international relevance: communications technology (e.g., social media and messaging services) has been used to spread misinformation and incite violence in numerous countries around the world, but also has enormous potential for conflict prevention (https://www.nature. com/articles/s41562-019-0587-5). This piece provides invaluable insight into a topic of global significance, but contributors such as Green Otunga, who are based outside the Global North, are hard to find in our pages.

Nature Human Behaviour is an international journal and we aspire to publish research, comment and opinion that are globally representative. We want the journal to reflect the full spectrum of knowledge creation and be a platform for voices from around the globe.

Currently, however, we are falling short of this ideal. In 2018, we received research manuscripts whose corresponding authors came from 51 different countries. But nearly half of our submissions (47\%) came from the US and the UK. And more than $80 \%$ of our submissions came from only 10 countries, all but one in the Global North (USA, UK, China, Germany, Netherlands, Japan, Australia, Canada, Israel, France). Our published content since we launched in 2017 reflects a similar imbalance, with only $11 \%$ of articles having corresponding authors outside the Global North. The same is true

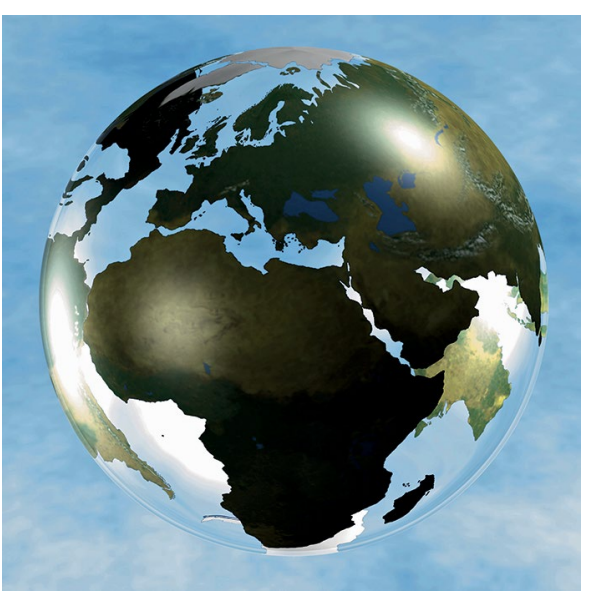

Credit: Cultura RM / Alamy Stock Photo

of our editorial team and our advisory panel: we are all based in the Global North.

We are committed to changing this. Although we have more limited control over our submissions, we have full control over who we invite to write for the journal, who we invite to review, who makes up our team and who we engage as advisors. To achieve the change we aspire to, we need your help.

Please write to us (humanbehaviour@ nature.com) to put forward yourself, a colleague, another expert or opinion leader outside the Global North as a potential contributor, reviewer or advisor. One of our key challenges once we have identified a potential contributor from an underrepresented country is finding a means of contacting them; including email addresses or other contact information with your recommendations is extremely helpful. If you are carrying out research on any aspect of individual or collective behaviour in an underrepresented country, contact us to discuss the suitability of your work for the journal. You can find the email addresses of all our editors here: https://www.nature. com/nathumbehav/about/editors. If we invite you to act as a reviewer, but you are unavailable, do let us know of other experts outside the Global North we can approach-we will be very grateful for it. We are currently looking for a new Associate or Senior Editor with a $\mathrm{PhD}$ in anthropology, ecology, evolution, epidemiology, public health or genetics to join our team (https:// career5.successfactors.eu/sfcareer/jobreqcare er?jobId $=25873 \&$ company $=$ C0001215517P \&username). Please help us spread the word; we will be thrilled to hear from applicants from the Global South.

We want Nature Human Behaviour to be a truly global journal. We are very grateful to everybody who has already contributed to the journal as an author, reviewer or advisor, regardless of geographical location. We are committed to working hard towards ensuring that this group becomes more geographically diverse, so that the journal can represent all of the international community, rather than just half of it.

Published online: 10 April 2019 https://doi.org/10.1038/s41562-019-0599-1 